\title{
MAGNONIC SPECTRA OF FERROMAGNETIC COMPOSITES VERSUS MAGNETIZATION CONTRAST
}

\author{
M. KrawczyK and H. Puszkarski* \\ Surface Physics Division, Institute of Physics, A. Mickiewicz University \\ Umultowska 85, 60-614 Poznań, Poland
}

(Received March 27, 1998)

\begin{abstract}
It had been shown recently that the calculated magnonic spectra of two-dimensional periodic ferromagnetic composites can present frequency ranges forbidden for the propagation of magnon excitations throughout the composite. However, those forbidden energy gaps were found to be highly sensitive to the exchange contrast between the component ferromagnetic materials but were very weakly sensitive to the contrast in spontaneous magnetizations of the two materials. Accordingly, in this paper we introduce a new mathematical definition of the exchange field acting in inhomogeneous rnedium. With this new definition the present theory gives magnonic spectra reasonably sensitive to magnetization contrast, as they should be from the physical viewpoint; moreover, the magnetization contrast now becomes a gap-creating factor as well.
\end{abstract}

PACS numbers: $75.50 . \mathrm{Gg}$

We consider a periodic structure composed of infinitely long cylinders made of a ferromagnetic material $A$ embedded in a ferromagnetic matrix $B$. The cylinders are assumed parallel to the $x_{3}$-axis of Cartesian coordinates leading to the existence of a two-dimensional periodic square lattice in the $\left(x_{1}, x_{2}\right)$-plane (Fig. 1a). The composite is acted on in the $x_{3}$-direction by a static magnetic field $\boldsymbol{H}_{0}$; the magnetization of the two materials $A$ and $B$ are parallel to $\boldsymbol{H}_{0}$. The lattice constant is denoted by $a$; the filling fraction $f$ is defined as the ratio of the area of the cross-section of a cylinder and that of the two-dimensional elementary cell. The ferromagnetic materials $A$ and $B$ are characterized by two quantities: their spontaneous magnetizations $M_{\mathrm{s}_{A}}$ and $M_{\mathrm{s}_{B}}$, and their exchange constants $A_{A}$ and $A_{B}$, both dependent on the position vector $x_{\|}=\left(x_{1}, x_{2}\right)$ lying in the plane perpendicular to the axis of the cylinders (whereas all these four quantities are homogeneous in the $x_{3}$-direction):

$$
M_{s}\left(x_{\|}\right)=M_{s_{B}}+\left(M_{s_{A}}-M_{s_{B}}\right) S\left(x_{\|}\right) \text {, }
$$

* Correspondence to: Dr. H. Puszkarski, Surface Physics Division, Institute of Physics, A. Mickiewicz University, Umultowska 85, Poznań,60-614 Poland. E-mail: henpusz@amu.edu.pl. 


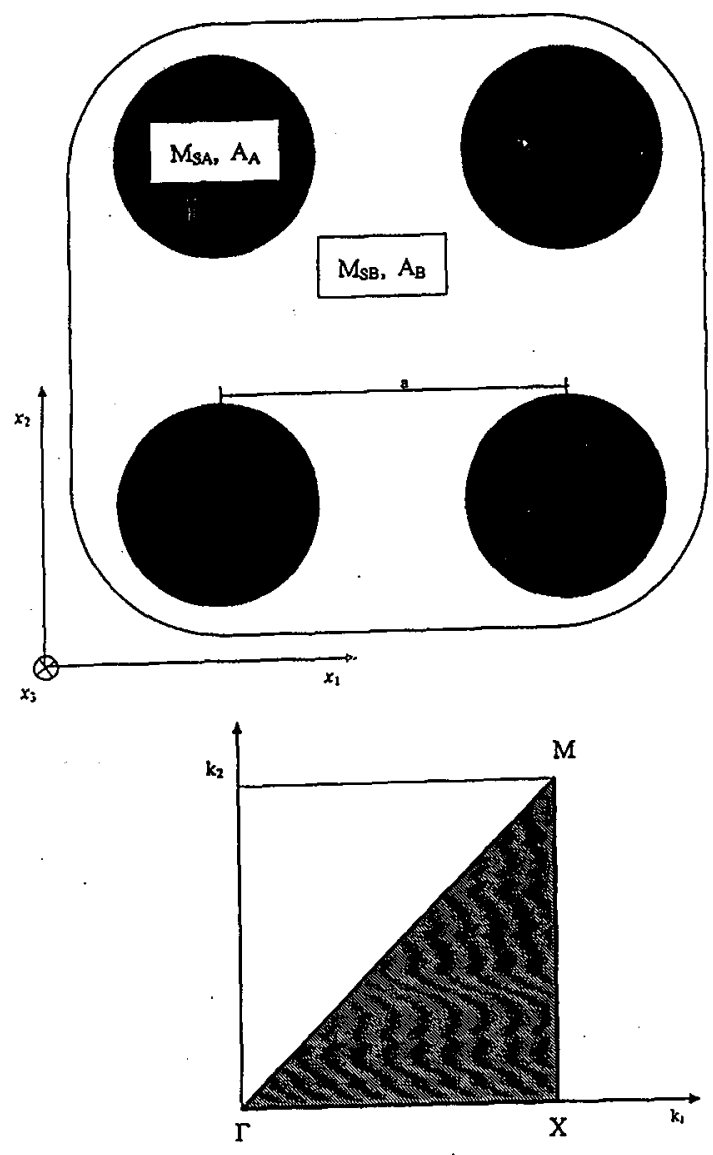

Fig. 1. (a) Elementary cell of the square lattice obtained after cross-section through a system of infinitely long cylinders (ferromagnet $A$ ) periodically embedded in an infinite matrix (ferromagnet $B$ ). (b) The first Brillouin zone of the square lattice and the path $\Gamma X M$, connecting the characteristic points for which the energy spectra are computed in Figs. 2a-d.

$$
A\left(x_{\|}\right)=A_{B}+\left(A_{\dot{A}}-A_{B}\right) S\left(x_{\|}\right)
$$

where the function $S\left(x_{\|}\right)$takes the value 1 inside the cylinders (in $A$ ) and 0 outside (in the surroundings $B$ ).

The Landau-Lifshits equation of motion in the inhomogeneous structure (on neglecting damping) is

$$
\frac{\partial}{\partial t} \boldsymbol{M}(\boldsymbol{r}, t)=\gamma \mu_{0} \boldsymbol{M}(\boldsymbol{r}, t) \times \boldsymbol{H}_{\mathrm{eff}}(r, t),
$$

where $\boldsymbol{M}(r, t)$ is the magnetization dependent on the three-dimensional position $r$ and time $t$. Here, $\boldsymbol{H}_{\text {eff }}(\boldsymbol{r}, t)$ stands for the effective magnetic field acting on the magnetization $M(r, t)$. 
Compared to Ref. [1] we propose a new (in our opinion more realistic) definition of the exchange field acting in inhomogeneous medium. The effective field is defined as follows:

$$
\boldsymbol{H}_{\mathrm{eff}}(r, t)=H_{0} e_{3}+h(r, t)+\frac{2}{\mu_{0}}\left(\nabla \cdot \frac{A}{M_{\mathrm{s}}^{2}} \nabla\right) \boldsymbol{M}(r, t),
$$

where $h(r, t)$ denotes the field of dipole interactions and the last right-hand term of (3) is the exchange field re-defined by us. It should be noted that the exchange field in the paper [1] by Vasseur et al. had been defined in the form

$$
\frac{2}{\mu_{0} M_{\mathrm{s}}}\left(\nabla \cdot \frac{A}{M_{\mathrm{s}}} \nabla\right) \boldsymbol{M}(\boldsymbol{r}, t)
$$

while the classical expression for the exchange field in homogeneous medium is of the well known form

$$
\frac{2 A}{\mu_{0} M_{\mathrm{s}}^{2}} \nabla^{2} \boldsymbol{M}(\boldsymbol{r}, t)
$$

In contradistinction to (4), our innovation consists in the inclusion of $M_{s}^{-2}$ to the right of $\nabla$. We search for a solution of Eq. (2) in a standard form, distinguishing in the magnetic moment a static part (along the $x_{3}$-axis) and a dynamic transversal part

$$
M(r, t)=\dot{M}_{\mathrm{s}} e_{3}+\boldsymbol{m}(r, t)
$$

the latter written as $m(r, t)$. We assume the time-dependence in the form

$$
\begin{aligned}
& m(r, t)=m(r) \mathrm{e}^{-\mathrm{i} \omega t}, \\
& h(r, t)=h(r) \mathrm{e}^{-\mathrm{i} \omega t},
\end{aligned}
$$

where $\omega$ is the circular frequency of the precession.

The dynamical dipole field has to fulfill the magnetostatic Maxwell equations

$$
\begin{aligned}
& \nabla \times h(r)=0, \\
& \nabla \cdot[h(r)+\lambda m(r)]=0
\end{aligned}
$$

( $\lambda=1$ in the SI units used by us). With respect to Eq. (8a), one introduces the magnetic potential $\psi$ :

$$
h(r)=-\nabla \psi(r)
$$

which, on insertion into Eq. (8b), gives an equation whence the potential $\psi$ can be determined

$$
\nabla^{2} \psi(r)-\lambda\left(\frac{\partial m^{x_{1}}(r)}{\partial x_{1}}+\frac{\partial m^{x_{2}}(r)}{\partial x_{2}}+\frac{\partial m^{x_{3}}(r)}{\partial x_{3}}\right)=0
$$

On insertion of (6), (7) and (3) into (2), and in a linear approximation (equivalent to assuming $m(r) \cdot e_{3}=0$ as done in [1]) we obtain our final equations: 


$$
\begin{aligned}
\mathrm{i} \Omega m^{x_{1}}(r)+\left[M_{\mathrm{s}} \boldsymbol{\nabla} \cdot Q \nabla\right] m^{x_{2}}(\boldsymbol{r})-m^{x_{2}}(\boldsymbol{r})-m^{x_{2}}(r)[\nabla \cdot Q \nabla] M_{\mathbf{s}} \\
+\frac{M_{\mathrm{s}}}{H_{0}} \frac{\partial \psi(\boldsymbol{r})}{\partial x_{2}}=0, \\
\mathrm{i} \Omega m^{x_{2}}(\boldsymbol{r})-\left[M_{\mathrm{s}} \boldsymbol{\nabla} \cdot Q \nabla\right] m^{x_{1}}(\boldsymbol{r})+m^{x_{1}}(\boldsymbol{r})+m^{x_{1}}(r)[\nabla \cdot Q \nabla] M_{\mathbf{s}} \\
\quad-\frac{M_{\mathrm{s}}}{H_{0}} \frac{\partial \psi(r)}{\partial x_{1}}=0,
\end{aligned}
$$

where we have introduced the notation

$$
\begin{aligned}
& \Omega \equiv \frac{\omega}{|\gamma| \mu_{0} H_{0}}, \\
& Q \equiv \frac{2 A}{\mu_{0} M_{\mathrm{s}}^{2} H_{0}} .
\end{aligned}
$$

Note now that our above derived equations differ from those of [1] by retaining the following terms, absent in Ref. [1]:

$$
-m^{x_{2}}(r)[\nabla \cdot Q \nabla] M_{\mathrm{s}}
$$

in Eq. (11a), and

$$
m^{x_{1}}(r)[\nabla \cdot Q \nabla] M_{\mathrm{s}}
$$

in Eq. (11b). The numerical treatment of Eqs. (11) taking place further on is similar to that of Ref. [1].

Figure 2 shows a comparison of the magnon spectra computed numerically with Eqs. (11) of the present paper and those computed with the respective formulae of [1]. The spin wave frequencies (in reduced units of $\Omega$ ) are plotted versus the reduced wave vector along the path $\Gamma X M$ of the first two-dimensional Brillouin zone (see Fig. 1b). Figure 2a shows the first 9 magnon branches obtained for a square lattice of Co cylinders embedded in Permalloy, computed with the equations of [1], whereas Fig. 2b shows the respective magnon band structure computed for the same composite and the same structural parameters albeit using Eqs. (11) of the present paper. Clearly, Fig. 2b shows energy gaps between the first and second band and between the fourth and fifth band, whereas the band structure of Fig. 2a shows no energy gaps altogether. Similarly, Figs. $2 c$ and $2 d$ show the respective magnon band structures of a composite consisting of $\mathrm{Fe}$ cylinders embedded in yttrium-iron garnet (YIG). The two structures differ essentially: whereas Fig. 2c fails to show gaps, we note that Fig. 2d exhibits three broad energy gaps between the first and the second band, between the third and fourth band, and between the seventh and eighth band. The existence of energy gaps in the cases (b) and (d) is due to the differences in spontaneous magnetization of the cylinders and their surroundings (the magnetization ratios in the cases considered above amount to, respectively: 1.7 for (Co/Permalloy) and 9.0 for ( $\mathrm{Fe} / \mathrm{YIG})$ ), since owing to the presence of the terms (14) and (15) our Eqs. (11) have become sensitive to the contrast in spontaneous magnetizations between the cylinders $A$ and the surroundings $B$, and consequently this contrast in spontaneous magnetizations strongly influences the conditions for the opening of energy gaps. 

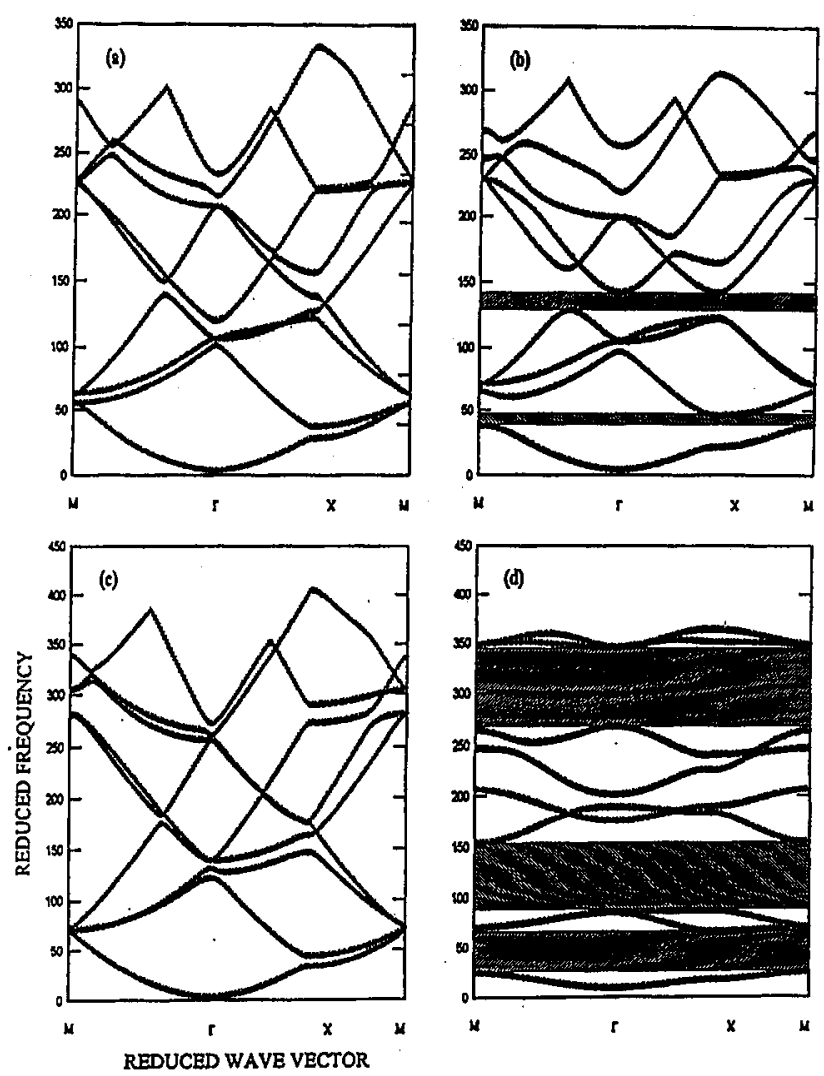

Fig. 2. Comparison of the magnonic bands computed after Ref. [1] (parts (a), (c)) and the bands computed with our Eqs. (11) (parts (b), (d)). Parameters assumed: filling fraction $f=0.5$, lattice constant $a=100 \AA$, and field $\mu_{0} H_{0}=0.1 \mathrm{~T}$. (a) Band structure computed for cobalt cylinders embedded in Permalloy - no gaps. (b) The structure as in (a), albeit computed with Eqs. (11) of the present paper - two energy gaps exist. (c) Band structure for iron cylinders embedded in yttrium-iron garnet - no gaps. (d) The same as in (c), but computed with Eqs. (11) of the present paper - three broad energy gaps exist.

To determine how the gap width depends on the spontaneous magnetization contrast, we shall consider a hypothetical composite in which the material parameters of the matrix (the surroundings) are maintained constant, whereas those of the cylinders are dealt with as variables. Figure 3 shows the width of the first gap (that between the first and second band) versus the exchange constant and spontaneous magnetization of the cylinders (embedded in a material with exchange constant $A=0.4 \times 10^{-11} \mathrm{~J} \mathrm{~m}^{-1}$ and spontaneous magnetization $M_{\mathrm{s}}=0.2 \times 10^{6} \mathrm{~A} \mathrm{~m}^{-1}$ ); the gap width is measured in units of the reduced frequency $\Omega$. The width of the energy gap is found to vary nonmonotonously with spontaneous magnetization of the cylinders (achieving one maximum), but, on the other hand, monotonously with growing exchange constant of the cylinders. 


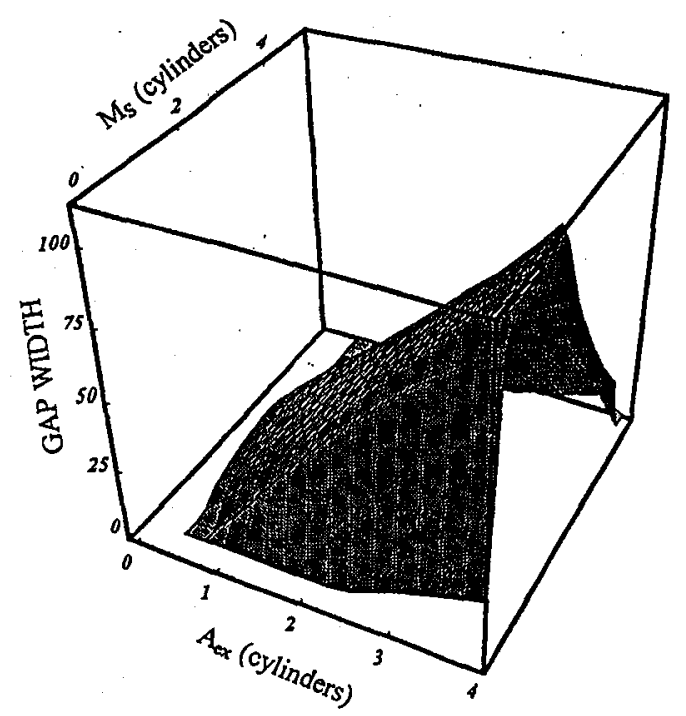

Fig. 3. The variability in width of the first energy gap (between the first and second band) versus the exchange constant and spontaneous magnetization of the cylinders. The cylinders are embedded in a material with characteristics close to those of YIG; the filling fraction $f=0.5$, lattice constant $a=100 \AA$, and field $\mu_{0} H_{0}=0.1 \mathrm{~T}$. The width achieves its maximum when the spontaneous magnetization of the cylinders becomes close to $2.2 \times 10^{6} \mathrm{~A} \mathrm{~m}^{-1}$.

We summarize: Our new definition of the exchange field acting in inhomogeneous medium resulted in lowering the contrast threshold required for the opening of gaps in the respective magnonic spectra; also, the existence conditions for the gaps are affected not only by the contrast in exchange constants (as it was the case with the former definition used in [1]) but also by the contrast in spontaneous magnetizations.

\section{Acknowledgments}

The present work was supported by grant No. 2P03B 04308 from the Committee for Scientific Research.

\section{References}

[1] J.O. Vasseur, L. Dobrzynski, B. Djafari-Rouhani, H. Puszkarski, Phys. Rev. B 54, 1043 (1996). 